\title{
LINEAR POLARIZATION OF RADIO FREQUENCY LINES IN MOLECULAR CLOUDS AND CIRCUMSTELLAR ENVELOPES
}

\author{
Peter Goldreich and Nikolaos D. Kylafis \\ California Institute of Technology \\ Received 1981 April 27; accepted 1981 August 18
}

\begin{abstract}
We predict that interstellar lines possess a few percent linear polarization provided that the optical depth in the source region is both anisotropic and of order unity and the radiative rates are at least comparable to the collision rates. These conditions are expected to be met in many sources which emit radio and far-infrared line radiation. Under circumstances in which the Zeeman splitting exceeds both the radiative and collisional rates the linear polarization is aligned either parallel or perpendicular to the projection of the magnetic field on the plane of the sky. This "strong magnetic field" limit is expected to apply to all radio frequency lines and to many of those far infrared lines which form between levels whose magnetic moments are comparable to the Bohr magneton. The "weak magnetic field" limit is relevant to most far-infrared lines formed between levels with magnetic moments of order the nuclear magneton. In this limit the polarization direction is determined by the orientation of the propagation direction with respect to the anisotropic optical depth.
\end{abstract}

Subject headings: interstellar: molecules - polarization - radio sources: lines stars: circumstellar shells - Zeeman effect

\section{INTRODUCTION}

Interstellar radio frequency lines provide a wealth of information about the physical conditions, spatial distribution, and kinematics of the interstellar gas. Polarization measurements would bear on the magnetic field direction, the optical depth, and the role of turbulence in the source. We expect linear polarization to occur for lines formed under conditions of moderate and anisotropic optical depth if radiative rates are competitive with collisional rates. The purpose of this paper is to evaluate the resultant polarization.

We consider a hypothetical molecule which possesses two rotational levels $a$ and $b$ with corresponding angular momenta $F_{a}=1$ and $F_{b}=0$. Level $a$ is split into three sublevels (denoted $+, 0,-$ ) in the presence of a magnetic field $\boldsymbol{B}=B_{0} \hat{\boldsymbol{b}}$. We take into account both radiative and collisional transitions between levels $a$ and $b$ as well as collisional relaxation among the sublevels of the upper level. Line formation is investigated in the limit that the systematic velocity differences are much greater than the thermal velocities of the molecules, a condition generally met in molecular clouds and circumstellar envelopes.

The calculations of polarization carried out in this paper could be easily extended to dipole transitions between levels with any $F_{a}$ and $F_{b}=F_{a} \pm 1$ by a simple application of the elegant formalism developed by Litvak (1975).

The plan of the paper is as follows. The equations describing the radiation field are derived in $\S$ II. In $\S$ III we set up the equation of motion of the molecular density matrix and discuss its solution in the strong and weak magnetic field limits. We derive the equations of radiative transfer for the Stokes parameters in $\S$ IV. The radiative transfer equations are solved using the Sobolev approximation in $\S \mathrm{V}$. Analytic expressions for the polarization in the limits of low and high optical depth are obtained in § VI. We discuss our results in § VII.

\section{THE ELECTROMAGNETIC FIELD}

We treat the radiation field classically and approximate it locally by a superposition of plane waves. The electric field $\boldsymbol{E}$ of the radiation induces microscopic dipole moments in the molecules. The macroscopic polarization $\boldsymbol{P}$ is obtained from the density matrix and acts as a source term in Maxwell's equations. Self-consistency requires that the 
assumed $\boldsymbol{E}$ field equal that derived from Maxwell's equations which in Gaussian units read

$$
\nabla \cdot D=4 \pi \rho, \quad \nabla \times E=-\frac{1}{c} \frac{\partial B}{\partial t}, \quad \nabla \cdot B=0, \quad \nabla \times B=\frac{1}{c} \frac{\partial D}{\partial t}, \quad D=E+4 \pi P,
$$

where we have neglected the free electron current density.

The wave equation for a plane wave traveling along $s \hat{n}$ is

$$
\frac{1}{c^{2}} \frac{\partial^{2} \boldsymbol{E}}{\partial t^{2}}+\hat{\boldsymbol{n}} \times\left(\hat{\boldsymbol{n}} \times \frac{\partial^{2} \boldsymbol{E}}{\partial s^{2}}\right)=-\frac{4 \pi}{c^{2}} \frac{\partial^{2} \boldsymbol{P}}{\partial t^{2}} .
$$

Following the work of Goldreich, Keeley, and Kwan (1973), we decompose the transverse part of the electric field into its circularly polarized components and write

$$
\boldsymbol{E}(s, t)=\operatorname{Re}\left[\mathrm{E}^{+}(s, t) \hat{\boldsymbol{e}}^{+}+\mathrm{E}^{-}(s, t) \hat{\boldsymbol{e}}^{-}\right],
$$

where

$$
\mathrm{E}^{ \pm}(s, t)=E^{ \pm}(s, t) \exp \left\{-i\left[\omega_{r}(t-s / c)+\varphi^{ \pm}(s, t)\right]\right\}
$$

and $\omega_{r}=2 \pi \nu_{r}$ is the resonant frequency of transitions between levels $a$ and $b$. The amplitudes $E^{ \pm}(s, t)$ and the phases $\varphi^{ \pm}(s, t)$ are real, slowly varying, functions of space and time. That is,

$$
\left|\frac{\partial E^{ \pm}}{\partial t}\right| \ll \omega_{r}\left|E^{ \pm}\right|, \quad \ll\left|\frac{\partial E^{ \pm}}{\partial s}\right| \ll k\left|E^{ \pm}\right|, \quad\left|\frac{\partial \varphi^{ \pm}}{\partial t}\right| \ll \omega_{r}, \quad\left|\frac{\partial \varphi^{ \pm}}{\partial s}\right| \ll k,
$$

where $k \hat{n}$ is the wave vector. A similar decomposition of the polarization yields

$$
\boldsymbol{P}(s, t)=\operatorname{Re}\left[\mathrm{P}^{+}(s, t) \hat{\boldsymbol{e}}^{+}+\mathrm{P}^{-}(s, t) \hat{\boldsymbol{e}}^{-}\right],
$$

where we write

$$
\mathrm{P}^{ \pm}(s, t)=P^{ \pm}(s, t) \exp \left\{-i\left[\omega_{r}(t-s / c)+\varphi^{ \pm}(s, t)\right]\right\} .
$$

Unlike $E^{ \pm}$which is real, $P^{ \pm}$is complex because the phase $\varphi^{ \pm}$is that of the electric field.

If we substitute equations (3), (5), and (6) into equation (2), project out the transverse components, and use inequalities (4), we get

$$
\frac{D}{D s}\left[E^{ \pm} \exp \left(-i \varphi^{ \pm}\right)\right]=i \frac{2 \pi \omega_{r}}{c} P^{ \pm} \exp \left(-i \varphi^{ \pm}\right)
$$

where

$$
\frac{D}{D s}=\frac{\partial}{\partial s}+\frac{1}{c} \frac{\partial}{\partial t}
$$

It follows immediately from equation (7) that

$$
\frac{D E^{ \pm 2}}{D s}=2 \pi i \frac{\omega_{r}}{c}\left[P^{ \pm} E^{ \pm}-P^{ \pm *} E^{ \pm}\right]
$$

and

$$
\frac{D}{D s}\left[E^{+} E^{-} \exp (-i \Delta \varphi)\right]=2 \pi i \frac{\omega_{r}}{c}\left[P^{+} E^{-}-P^{-*} E^{+}\right] \exp (-i \Delta \varphi),
$$

where $\Delta \varphi=\varphi^{+}-\varphi^{-}$. Equations (8) and (9) govern the transfer of polarized radiation. The next three sections are devoted to evaluating the source terms in these equations. 
Polarized radiation is conveniently described by the Stokes parameters $I(\nu), Q(\nu), V(\nu)$, and $U(\nu)$ (Chandrasekhar 1950):

$$
\begin{array}{llrl}
\int_{-\infty}^{\infty} I(\nu) d \nu & =\frac{c}{8 \pi}\left\langle E^{+2}+E^{-2}\right\rangle, & \int_{-\infty}^{\infty} V(\nu) d \nu & =\frac{c}{8 \pi}\left\langle E^{+2}-E^{-2}\right\rangle, \\
\int_{-\infty}^{\infty} Q(\nu) d \nu & =\frac{c}{8 \pi}\left\langle 2 E^{+} E^{-} \cos \Delta \varphi\right\rangle, & \int_{-\infty}^{\infty} U(\nu) d \nu & =\frac{c}{8 \pi}\left\langle 2 E^{+} E^{-} \sin \Delta \varphi\right\rangle,
\end{array}
$$

where the angular brackets denote expectation value.

\section{THE DENSITY MATRIX EQUATION OF MOTION}

The quantum mechanical behavior of the molecules is described by means of the density matrix $\rho$ which is a function of position, velocity, and time. Our discussion and approximate solution of the equation of motion for $\rho$ follows that of Goldreich, Keeley, and Kwan (1973).

The equation of motion satisfied by the density matrix is

$$
\frac{\partial \rho}{\partial t}=-i[H \rho-\rho H]+\mathrm{A}+\mathrm{C} .
$$

Here $H$ is the semiclassical Hamiltonian which governs stimulated emission and absorption, A accounts for spontaneous emission, and $\mathrm{C}$ describes collisions.

The macroscopic velocity field at each point in the source is, for our purposes, characterized by the eigenvectors $\Lambda_{1} \hat{i}$, $\Lambda_{2} \hat{j}, \Lambda_{3} \hat{\boldsymbol{k}}$ of the symmetric part of the rate-of-strain tensor. The antisymmetric part of this tensor is irrelevant because it represents rotation which plays no role in Doppler shifts. We choose the three unit eigenvectors as coordinate axes with $\hat{\boldsymbol{k}}$ the polar axis. The propagation direction $\hat{\boldsymbol{n}}$ is described by the polar angle $\boldsymbol{\vartheta}$ and the angle $\lambda$ between $\hat{\boldsymbol{i}}$ and $(\hat{k} \times \hat{n}) /|\hat{k} \times \hat{n}|$. Thus $\hat{\boldsymbol{n}}=(\sin \vartheta \sin \lambda, \sin \vartheta \cos \lambda, \cos \vartheta)$.

The density matrix equation of motion (11) will be discussed in the two limiting cases of strong and weak magnetic field. In both cases we assume that the Zeeman splitting is much smaller than the total Doppler width $\Delta \omega$ so that circular polarization does not arise. Even if the Zeeman splitting were greater than the thermal Doppler width, our calculations for the linear polarization would be valid. The strong field limit applies where the Zeeman splitting is much larger than the collision rate and the spontaneous and stimulated radiative transition rates. The conditions under which interstellar millimeter lines form generally fall in the strong field case even for molecules whose magnetic moments are comparable to the nuclear magneton. However, for submillimeter lines formed in high density regions, the spontaneous emission and collision rates could be much larger than the Zeeman splitting, and the weak field limit would apply.

In Paper I (Goldreich and Kylafis 1981) we discussed the strong field limit and used the rate equations. In this paper the emphasis is on the weak field limit where the rate equations are not valid, but the strong field limit is presented as well for completeness and comparison.

Before examining each limiting case separately, we make the density matrix equation of motion (11) more transparent. We define $\Gamma$ and $\Lambda$ by

$$
\mathrm{A}+\mathrm{C}=-\frac{1}{2}[\Gamma \rho+\rho \Gamma]+\Lambda .
$$

The anticommutator in equation (12) describes decays while $\Lambda$ describes excitations. Both $\Gamma$ and $\Lambda$ are diagonal matrices. Their elements read

$$
\begin{aligned}
\Gamma_{b b} & =3 C \exp \left(\frac{h \nu_{r}}{k T}\right), & \Gamma_{m m} & =C+3 C^{\prime}+A, \\
\Lambda_{b b} & =C \sum_{n} \rho_{n n}, & \Lambda_{m m} & =C \rho_{b b} \exp \left(\frac{h \nu_{r}}{k T}\right)+C^{\prime} \sum_{n} \rho_{n n},
\end{aligned}
$$

where $m$ and $n$ refer to the sublevels $(+, 0,-)$ of the $a$ level, $C$ is the collision rate for transitions between levels $a$ and $b, C^{\prime}$ is the collision rate for transitions between sublevels of level $a, A$ is the spontaneous emission rate from the upper sublevels, and $T$ is the kinetic temperature. 
The matrix elements of the Hamiltonian $H$ are

$$
H_{m n}=\left(\omega_{r}+\omega_{g} m\right) \delta_{m n}, \quad H_{b b}=0, \quad H_{m b}=V_{m b},
$$

where $V_{m b}$ is a linear combination of the components of $\boldsymbol{E}, \omega_{g}=\mu B_{0} / \hbar$, and $\mu$ is the magnetic moment of the molecules.

We make use of the rotating wave approximation (Lamb 1964; Sargent, Scully, and Lamb 1974) in which only terms proportional to $\exp \left(-i \omega_{r} t\right)$ are retained. Thus

$$
\boldsymbol{E}=\frac{1}{2}\left\{E^{+} \exp \left(-i \varphi^{+}\right) \hat{\boldsymbol{e}}^{+}+E^{-} \exp \left(-i \varphi^{-}\right) \hat{\boldsymbol{e}}^{-}\right\} \exp \left[-i \omega_{r}(t-s / c)\right] .
$$

Since $\rho$ is a function of velocity, we write

$$
\rho=\bar{\rho} \varphi_{v},
$$

where $\varphi_{v}$ is the velocity profile function such that

$$
\bar{\rho} \equiv \int_{-\infty}^{\infty} d v \rho \text { and } \int_{-\infty}^{\infty} \varphi_{v} d v=1
$$

It should be noted here that equation (16) is not strictly correct, because each component of the density matrix has its own velocity profile function. Nevertheless, in what follows we use equation (16) and only remark that our arguments go through in the more general case as well. For notational convenience we drop the bar above $\rho$ from here on. In component form, the density matrix equation of motion reads:

$$
\begin{aligned}
& \frac{\partial \rho_{m m}}{\partial t}=-i \int d \Omega \int_{-\infty}^{\infty} \varphi_{v} d v\left(V_{m b} \rho_{b m}-\rho_{m b} V_{b m}\right)-\Gamma_{m m} \rho_{m m}+C \exp \left(-\frac{h \nu_{r}}{k T}\right) \rho_{b b}+C^{\prime} \sum_{n} \rho_{n n}, \\
& \frac{\partial \rho_{m n}}{\partial t}=-i \omega_{g}(m-n) \rho_{m n}-i \int d \Omega \int_{-\infty}^{\infty} \varphi_{v} d v\left(V_{m b} \rho_{b n}-\rho_{m b} V_{b n}\right)-\Gamma_{m m} \rho_{m n}, \\
& \frac{\partial \rho_{m b}}{\partial t}=-i \int d \Omega \int_{-\infty}^{\infty} \varphi_{v} d v\left[V_{m b} \rho_{b b}+\left(\omega_{r}+\omega_{g} m\right) \rho_{m b}\right]+i \int d \Omega \int \varphi_{v} d v \sum_{n} \rho_{m n} V_{n b}-\Gamma \rho_{m b},
\end{aligned}
$$

where $\Gamma=\left(\Gamma_{b b}+\Gamma_{m m}\right) / 2$. Assuming $\rho_{m m}$ and $\rho_{m n}$ vary only slightly on a time scale $\Delta \omega^{-1}$, a formal solution of equation (20) yields

$$
\begin{aligned}
\rho_{m b}= & i \sum_{n} \rho_{m n} \int d \Omega \int_{-\infty}^{\infty} \varphi_{v} d v \int_{-\infty}^{t} \exp \left\{-\left[\Gamma+i\left(\omega_{r}+\omega_{g} m\right)\right]\left(t-t^{\prime}\right)\right\} V_{n b}^{\prime} d t^{\prime} \\
& -i \rho_{b b} \int d \Omega \int_{-\infty}^{\infty} \varphi_{v} d v \int_{-\infty}^{t} \exp \left\{-\left[\Gamma+i\left(\omega_{r}+\omega_{g} m\right)\right]\left(t-t^{\prime}\right)\right\} V_{m b}^{\prime} d t^{\prime}
\end{aligned}
$$

A prime attached to $V$ indicates that its argument is $\left(s^{\prime}, t^{\prime}\right)$ where $s^{\prime}=s-v\left(t-t^{\prime}\right)$.

Substituting equations (21) into equations (18) and (19) and taking the expectation values of the resulting expressions, we get

$$
\begin{aligned}
\frac{\partial \rho_{m m}}{\partial t}= & \left(\rho_{b b}-\rho_{m m}\right) 2 \operatorname{Re} \int d \Omega \int_{-\infty}^{\infty} \varphi_{v} d v \int_{-\infty}^{t} \exp \left\{-\left[\Gamma+i\left(\omega_{r}+\omega_{g} m\right)\right]\left(t-t^{\prime}\right)\right\}\left\langle V_{m b}^{\prime} V_{b m}\right\rangle d t^{\prime} \\
& -\sum_{n \neq m} 2 \operatorname{Re} \rho_{m n} \int d \Omega \int_{-\infty}^{\infty} \varphi_{v} d v \int_{-\infty}^{t} \exp \left\{-\left[\Gamma+i\left(\omega_{r}+\omega_{g} m\right)\right]\left(t-t^{\prime}\right)\right\}\left\langle V_{n b}^{\prime} V_{b m}\right\rangle d t^{\prime} \\
& +C \exp \left(-\frac{h \nu_{r}}{k T}\right) \rho_{b b}+C^{\prime} \sum_{n} \rho_{n n}-\left(C+3 C^{\prime}+A\right) \rho_{m m}
\end{aligned}
$$


Since $\rho$ is a function of velocity, we write

$$
\begin{aligned}
\frac{\partial \rho_{m n}}{\partial t}= & \left(\rho_{b b}-\rho_{n n}\right) \int d \Omega \int_{-\infty}^{\infty} \varphi_{v} d v \int_{-\infty}^{t} \exp \left\{-\left[\Gamma-i\left(\omega_{r}+\omega_{g} n\right)\right]\left(t-t^{\prime}\right)\right\}\left\langle V_{n b}^{\prime} V_{b m}\right\rangle^{*} d t^{\prime} \\
& +\left(\rho_{b b}-\rho_{m m}\right) \int d \Omega \int_{-\infty}^{\infty} \varphi_{v} d v \int_{-\infty}^{t} \exp \left\{-\left[\Gamma+i\left(\omega_{r}+\omega_{g} m\right)\right]\left(t-t^{\prime}\right)\right\}\left\langle V_{m b}^{\prime} V_{b n}\right\rangle d t^{\prime} \\
& -\sum_{k \neq n} \rho_{n k} * \int d \Omega \int_{-\infty}^{\infty} \varphi_{v} d v \int_{-\infty}^{t} \exp \left\{-\left[\Gamma-i\left(\omega_{r}+\omega_{g} n\right)\right]\left(t-t^{\prime}\right)\right\}\left\langle V_{k b}^{\prime} V_{b m}\right\rangle^{*} d t^{\prime} \\
& -\sum_{k \neq m} \rho_{m k} \int d \Omega \int_{-\infty}^{\infty} \varphi_{v} d v \int_{-\infty}^{t} \exp \left\{-\left[\Gamma+i\left(\omega_{r}+\omega_{g} m\right)\right]\left(t-t^{\prime}\right)\right\}\left\langle V_{k b}^{\prime} V_{b n}\right\rangle d t^{\prime} \\
& -\left[\left(C+3 C^{\prime}+A\right)+i \omega_{g}(m-n)\right] \rho_{m n} .
\end{aligned}
$$

We rewrite equations (22) and (23) as

$$
\begin{aligned}
\frac{\partial \rho_{m m}}{\partial t}= & R_{m m}\left(\rho_{b b}-\rho_{m m}\right)-\sum_{n \neq m} \operatorname{Re}\left(R_{n m} \rho_{m n}\right)+C \exp \left(-\frac{h \nu_{r}}{k T}\right) \rho_{b b}+C^{\prime} \sum_{n} \rho_{n n}-\left(C+3 C^{\prime}+A\right) \rho_{m m}, \\
\frac{\partial \rho_{m n}}{\partial t}= & \frac{1}{2} R_{n m}^{*}\left(\rho_{b b}-\rho_{n n}\right)+\frac{1}{2} R_{m n}\left(\rho_{b b}-\rho_{m m}\right)-\sum_{k \neq n} \frac{1}{2} R_{k m}^{*} \rho_{n k}^{*}-\sum_{k \neq m} \frac{1}{2} R_{k n} \rho_{m k} \\
& -\left[\left(C+3 C^{\prime}+A\right)+i \omega_{g}(m-n)\right] \rho_{m n},
\end{aligned}
$$

where we have defined the rates $R_{m m}$ and $R_{m n}$ to be

$$
\begin{aligned}
R_{m m} & =2 \operatorname{Re} \int d \Omega \int_{-\infty}^{\infty} \varphi_{v} d v \int_{-\infty}^{t} \exp \left\{-\left[\Gamma+i\left(\omega_{r}+\omega_{g} m\right)\right]\left(t-t^{\prime}\right)\right\}\left\langle V_{m b}^{\prime} V_{b m}\right\rangle d t^{\prime}, \\
R_{m n} & =\int d \Omega \int_{-\infty}^{\infty} \varphi_{v} d v \int_{-\infty}^{t} \exp \left\{-\left[\Gamma+i\left(\omega_{r}+\omega_{g} m\right)\right]\left(t-t^{\prime}\right)\right\}\left\langle V_{m b}^{\prime} V_{b n}\right\rangle d t^{\prime}
\end{aligned}
$$

We assume that the radiation field has stationary statistical properties. Thus expectation values are equivalent to time averages. If the electric field is written as a Fourier integral

$$
E^{\mu}(s, t)=(2 \pi)^{-1 / 2} \int_{-\infty}^{\infty} E^{\mu}(\omega) \exp [-i \omega(t-s / c)] d \omega, \quad \mu=+,-,
$$

then

$$
\left\langle E^{\mu}(\omega) E^{\nu *}\left(\omega^{\prime}\right)\right\rangle=2 \pi F^{\mu \nu}(\omega) \delta\left(\omega-\omega^{\prime}\right),
$$

where the angular brackets denote expectation value. Using equations (28) and (29), one can show that

$$
\left\langle E^{\mu}(s, t) E^{\nu *}\left(s^{\prime}, t^{\prime}\right)\right\rangle=\int_{-\infty}^{\infty} d \omega F^{\mu \nu}(\omega) \exp \left[-i \omega\left(1-\frac{v}{c}\right)\left(t-t^{\prime}\right)\right],
$$

which is enough to describe the expectation values that appear in equations (26) and (27). Equations (26) and (27) involve integrals of the form

$$
\int_{-\infty}^{\infty} \varphi_{v} d v \int_{-\infty}^{t} \exp \left\{-\left[\Gamma+i\left(\omega_{r}+\omega_{g} m\right)\right]\left(t-t^{\prime}\right)\right\}\left\langle E^{\mu}(s, t) E^{\nu *}(s, t)\right\rangle d t^{\prime}
$$

Integrating first over time and then over frequency and making the well justified approximation that $\Gamma \ll \Delta \omega$, we 


$$
\pi \int_{-\infty}^{\infty} \varphi_{\omega} d \omega F^{\mu \nu}(\omega)
$$

where $\varphi_{\omega}$ is the normalized radian frequency profile function. We define the quantities $U_{m n}(\omega)$ by

$$
\left\langle V_{m b}^{\prime} V_{b n}\right\rangle=\int_{-\infty}^{\infty} d \omega U_{m n}(\omega) \exp \left\{-i \omega(1-v / c)\left(t-t^{\prime}\right)\right\}
$$

Following the same procedure as for expressions (31), we write equations (26) and (27) as

$$
\begin{gathered}
R_{m m}=2 \pi \int d \Omega \int d \omega \varphi_{\omega} U_{m m}(\omega), \\
R_{m n}=\pi \int d \Omega \int d \omega \varphi_{\omega} U_{m n}(\omega) .
\end{gathered}
$$

Since the Zeeman splitting is assumed to be much smaller than the line width, circular polarization does not arise and $\rho_{++}=\rho_{--} \equiv \rho_{ \pm \pm}$.

Equations (24) and (25) will be discussed in two limiting cases according to the relative values of the Zeeman splitting and the collision and radiative rates.

\section{a) Case 1: Strong Field Limit}

It is easy to show mathematically that in this limit the off-diagonal elements $\rho_{m n}$ of the density matrix are negligible. In steady state, a first order approximation to $\rho_{m n}$ is obtained from equation (25) which reads

$$
0 \approx-i \omega_{g}(m-n) \rho_{m n}+\frac{1}{2} R_{m n}\left(\rho_{b b}-\rho_{n n}\right)+\frac{1}{2} R_{m n}\left(\rho_{b b}-\rho_{m m}\right) .
$$

We see that $\rho_{m n}$ is much smaller than $\left(\rho_{b b}-\rho_{k k}\right)$ and can therefore be neglected in equation (24). With $\rho_{m n}=0$, the density matrix equation of motion reduces to the rate equations

$$
\frac{\partial \rho_{m m}}{\partial t}=R_{m m}\left(\rho_{b b}-\rho_{m m}\right)-A \rho_{m m}+C \exp \left(-\frac{h \nu_{r}}{k T}\right) \rho_{b b}+C^{\prime} \sum_{n} \rho_{n n}-\left(C+3 C^{\prime}\right) \rho_{m m},
$$

which were used in Paper I.

\section{b) Case 2: Weak Field Limit}

In the weak field limit the rate equations (34) are not valid. The off-diagonal elements $\rho_{m n}$ cannot be neglected in the equation of motion for the diagonal elements $\rho_{m m}$. Therefore we must solve equations (24) and (25) simultaneously. Symmetry arguments allow us to assume that $\rho_{ \pm 0}=0, \rho_{+-}$is real, and $R_{ \pm 0}=0$. Equations (24) and (25) reduce to

$$
\begin{aligned}
\frac{\partial \rho_{ \pm \pm}}{\partial t} & =R_{ \pm \pm}\left(\rho_{b b}-\rho_{ \pm \pm}\right)-R_{+-} \rho_{+-}-A \rho_{ \pm \pm}+C\left[\rho_{b b} \exp \left(-\frac{h \nu_{r}}{k T}\right)-\rho_{ \pm \pm}\right]-C^{\prime}\left(\rho_{ \pm \pm}-\rho_{00}\right), \\
\frac{\partial \rho_{00}}{\partial t} & =R_{00}\left(\rho_{b b}-\rho_{00}\right)-A \rho_{00}+C\left[\rho_{b b} \exp \left(-\frac{h \nu_{r}}{k T}\right)-\rho_{00}\right]-2 C^{\prime}\left(\rho_{00}-\rho_{ \pm \pm}\right), \\
\frac{\partial \rho_{+-}}{\partial t} & =R_{+-}\left(\rho_{b b}-\rho_{ \pm \pm}\right)-R_{ \pm \pm} \rho_{+-}-A \rho_{+-}-\left(C+3 C^{\prime}\right) \rho_{+-} .
\end{aligned}
$$

\section{THE EQUATIONS OF RADIATIVE TRANSFER}

\section{a) Case 1: Strong Field Limit}

In the limit of strong magnetic field, the field direction provides a natural choice for the axis of quantization. We choose polarization vectors $\hat{\boldsymbol{e}}_{\perp}$ and $\hat{\boldsymbol{e}}_{\|}=\hat{\boldsymbol{n}} \times \hat{\boldsymbol{e}}_{\perp}$ such that the third Stokes parameter $U$ is zero. This requirement is 
used (later) to prove that the angle $\eta$ between $\hat{\boldsymbol{e}}_{\perp}$ and $\hat{\boldsymbol{\varepsilon}} \equiv(\hat{\boldsymbol{b}} \times \hat{\boldsymbol{n}}) /|\hat{\boldsymbol{b}} \times \hat{\boldsymbol{n}}|$ vanishes. The circularly polarized unit vectors are given by $\hat{\boldsymbol{e}}^{ \pm}=2^{-1 / 2}\left(\hat{\boldsymbol{e}}_{\perp} \pm i \hat{\boldsymbol{e}}_{\|}\right)$.

The matrix elements $V_{m b}$ are

$$
V_{m b}(s, t)=\frac{d}{\hbar}\left\{\boldsymbol{E} \cdot \frac{\left(\hat{\boldsymbol{\varepsilon}}-i \hat{\boldsymbol{\varepsilon}}^{\prime}\right)}{\sqrt{2}} \boldsymbol{\delta}_{m+}-\boldsymbol{E} \cdot \hat{b} \boldsymbol{\delta}_{m 0}-\boldsymbol{E} \cdot \frac{\left(\hat{\boldsymbol{\varepsilon}}+i \hat{\boldsymbol{\varepsilon}}^{\prime}\right)}{\sqrt{2}} \boldsymbol{\delta}_{m-}\right\}
$$

where $d$ is the reduced dipole matrix element of the transition (Sargent, Lamb, and Fork 1967). The unit vector $\hat{\boldsymbol{\varepsilon}}^{\prime}$ is defined by $\hat{\boldsymbol{\varepsilon}}^{\prime}=\hat{\boldsymbol{b}} \times \hat{\boldsymbol{\varepsilon}}$, such that $\left(\hat{\boldsymbol{\varepsilon}}, \hat{\boldsymbol{\varepsilon}}^{\prime}, \hat{\boldsymbol{b}}\right)$ form a right-handed system. With the above definitions the following relations hold:

$$
\begin{aligned}
\frac{\left(\hat{\boldsymbol{\varepsilon}} \pm i \hat{\boldsymbol{\varepsilon}}^{\prime}\right)}{\sqrt{2}} & =\frac{(1 \pm \cos \gamma)}{2} \exp (i \eta) \hat{\boldsymbol{e}}^{+}+\frac{(1 \mp \cos \gamma)}{2} \exp (-i \eta) \hat{\boldsymbol{e}}^{-} \mp \frac{\sin \gamma}{\sqrt{2}} \hat{\boldsymbol{n}} \\
\hat{\boldsymbol{b}} & =\hat{\boldsymbol{n}} \cos \gamma-i \frac{\sin \gamma}{\sqrt{2}}\left[\exp (i \eta) \hat{\boldsymbol{e}}^{+}-\exp (-\eta) \hat{\boldsymbol{e}}^{-}\right]
\end{aligned}
$$

where $\gamma$ is the angle between $\hat{\boldsymbol{n}}$ and $\hat{\boldsymbol{b}}$.

To derive expressions for the radiative rates $U_{ \pm \pm}, U_{00}, U_{ \pm \mp}$, and $U_{ \pm 0}$ we use definition (32) with $t=t^{\prime}$ and equations (10), (36), and (37). We obtain:

$$
\begin{aligned}
U_{ \pm \pm}(\omega) & =\frac{\pi}{2 c}\left(\frac{d}{\hbar}\right)^{2}\left\{\left(1+\cos ^{2} \gamma\right) I(\omega) \pm 2 \cos \gamma V(\omega)+\sin ^{2} \gamma[\cos 2 \eta Q(\omega)-\sin 2 \eta U(\omega)]\right\} \\
U_{00}(\omega) & =\frac{\pi}{c}\left(\frac{d}{\hbar}\right)^{2} \sin ^{2} \gamma[I(\omega)-\cos 2 \eta Q(\omega)+\sin 2 \eta U(\omega)],
\end{aligned}
$$

$U_{ \pm \mp}(\omega)=-\frac{\pi}{2 c}\left(\frac{d}{\hbar}\right)^{2}\left\{\sin ^{2} \gamma I(\omega)+\left(1+\cos ^{2} \gamma\right)[\cos 2 \eta Q(\omega)-\sin 2 \eta U(\omega)] \mp i \cos \gamma[\sin 2 \eta Q(\omega)+\cos 2 \eta U(\omega)]\right\}$

$U_{ \pm 0}(\omega)= \pm i \frac{\pi}{c \sqrt{2}}\left(\frac{d}{\hbar}\right)^{2} \sin \gamma\{V(\omega) \pm \cos \gamma I(\omega)+[i \sin 2 \eta \mp \cos \gamma \cos 2 \eta] Q(\omega)+[i \cos 2 \eta \pm \cos \gamma \sin 2 \eta] U(\omega)\}$

Since circular polarization does not arise in our problems, $V=0$ and $U_{++}=U_{--}$. Expressions for the spontaneous emission rates $A_{ \pm \pm}\left(\omega_{r}\right)$ and $A_{00}\left(\omega_{r}\right)$ follow from equations (38) if we regard spontaneous emission as emission induced by the zero-point fluctuations for which

$$
I\left(\omega_{r}\right)=I_{0}\left(\omega_{r}\right)=\frac{2 h \omega_{r}^{3}}{(2 \pi)^{3} c^{2}}, \quad \text { and } \quad V(\omega)=Q(\omega)=U(\omega)=0
$$

We find

$$
\begin{aligned}
A_{ \pm \pm}\left(\omega_{r}\right) & =\frac{d^{2} \omega_{r}^{3}}{2 h c^{3}}\left(1+\cos ^{2} \gamma\right), \\
A_{00}\left(\omega_{r}\right) & =\frac{d^{2} \omega_{r}^{3}}{h c^{3}} \sin ^{2} \gamma
\end{aligned}
$$


Integrating (39a) and (39b) over all angles, we get the total spontaneous emission rate $A$ from one sublevel:

$$
A=\frac{8 \pi}{3} \frac{d^{2} \omega_{r}^{3}}{h c^{3}}
$$

In order to write down the equations of radiative transfer, we must first evaluate the molecular source terms that appear in equations (8) and (9). The macroscopic polarization $\boldsymbol{P}(s, t)$ induced in the medium by the radiation field is given by

$$
\boldsymbol{P}(s, t)=\int_{-\infty}^{\infty} \operatorname{tr}(\rho e r) \varphi_{v} d v
$$

where $\boldsymbol{r}$ is the matrix of the position vector. The nonvanishing matrix elements of the dipole moment operator $\boldsymbol{d}=\boldsymbol{e r}$ are given by

$$
d_{ \pm b}=\mp d \frac{\left(\hat{\boldsymbol{\varepsilon}} \mp i \hat{\boldsymbol{\varepsilon}}^{\prime}\right)}{\sqrt{2}}, \quad d_{0 b}=d \hat{\boldsymbol{b}} .
$$

Using equations (6), (37), (40), (41), and the rotating wave approximation for $\boldsymbol{P}$, we get

$$
\begin{aligned}
P^{ \pm}=2 d \int_{-\infty}^{\infty} \varphi_{v} d v & {\left[-\frac{(1 \pm \cos \gamma)}{2} \rho_{+b} \mp i \frac{\sin \gamma}{\sqrt{2}} \rho_{0 b}+\frac{(1 \mp \cos \gamma)}{2} \rho_{-b}\right.} \\
& \left.-\frac{(1 \mp \cos \gamma)}{2} \rho_{+b}^{*} \mp i \frac{\sin \gamma}{\sqrt{2}} \rho_{0 b}^{*}+\frac{(1 \pm \cos \gamma)}{2} \rho_{-b}^{*}\right] \exp \left[i \omega_{r}\left(t-\frac{s}{c}\right)+i \varphi^{ \pm} \pm i \eta\right] .
\end{aligned}
$$

Equations (3), (15), (36), and (37) enable us to express $E^{ \pm}$in terms of the $V$ 's. We obtain

$$
E^{ \pm}=\left(\frac{\hbar}{d}\right)\left(V_{+b}-V_{-b} \pm i \frac{\sqrt{2}}{\sin \gamma} V_{0 b}\right) \exp \left[i \omega_{r}\left(t-\frac{s}{c}\right)+i \varphi^{ \pm} \mp i \eta\right]
$$

The equations obeyed by the Stokes parameters $I(\omega), Q(\omega)$, and $U(\omega)$ are obtained from equations (8), (9), (10), (21), (38), (42), and (43), and read

$$
\begin{aligned}
& \frac{D I}{D s}=-\frac{4 \pi^{3} d^{2} \omega_{r} \varphi_{\omega}}{h c}\left\{\left(\rho_{b b}-\rho_{ \pm \pm}\right)\left[\left(1+\cos ^{2} \gamma\right) I+\sin ^{2} \gamma(\cos 2 \eta Q-\sin 2 \eta U)\right]\right. \\
& \left.+\left(\rho_{b b}-\rho_{00}\right) \sin ^{2} \gamma[I-(\cos 2 \eta Q-\sin 2 \eta U)]-\left[\rho_{ \pm \pm}\left(1+\cos ^{2} \gamma\right)+\rho_{00} \sin ^{2} \gamma\right] I_{0}\right\} . \\
& \frac{D Q}{D s}=-\frac{4 \pi^{3} d^{2} \omega_{r} \varphi_{\omega}}{h c}\left\{\left(\rho_{b b}-\rho_{ \pm \pm}\right)\left[\left(1+\cos ^{2} \gamma\right) Q+\cos 2 \eta \sin ^{2} \gamma I\right]\right. \\
& \left.+\left(\rho_{b b}-\rho_{00}\right) \sin ^{2} \gamma(Q-\cos 2 \eta I)-\left(\rho_{ \pm \pm}-\rho_{00}\right) \cos 2 \eta \sin ^{2} \gamma I_{0}\right\} . \\
& \frac{D U}{D s}=-\frac{4 \pi^{3} d^{2} \omega_{r} \varphi_{\omega}}{h c}\left\{\left(\rho_{b b}-\rho_{ \pm \pm}\right)\left[\left(1+\cos ^{2} \gamma\right) U-\sin 2 \eta \sin ^{2} \gamma I\right]\right. \\
& \left.+\left(\rho_{b b}-\rho_{00}\right) \sin ^{2} \gamma[U+\sin 2 \eta I]+\left(\rho_{ \pm \pm}-\rho_{00}\right) \sin 2 \eta \sin ^{2} \gamma I_{0}\right\} .
\end{aligned}
$$

The terms proportional to $I_{0}$ are due to spontaneous emission.

The angle $\eta$ is to be determined so that $U=0$. The terms in $D U / D$ s proportional to either $I$ or $I_{0}$ and the terms in $D I / D$ s proportional to $U$ are zero if $\sin 2 \eta=0$. Taking $-\pi / 2<\eta \leq \pi / 2$, we conclude that in the strong field limit $\eta=0$ or $\eta=\pi / 2$. Thus the polarization is either parallel or perpendicular to $[\hat{\boldsymbol{b}}-(\hat{\boldsymbol{b}} \cdot \hat{\boldsymbol{n}}) \hat{\boldsymbol{n}}]$. 
The radiative transfer equations (44) reduce to the following:

$$
\begin{aligned}
\frac{D I}{D s}=-\frac{4 \pi^{3} d^{2} \omega_{r} \varphi_{\omega}}{h c}\{ & {\left[\left(\rho_{b b}-\rho_{ \pm \pm}\right)\left(1+\cos ^{2} \gamma\right)+\left(\rho_{b b}-\rho_{00}\right) \sin ^{2} \gamma\right] I-\left(\rho_{ \pm \pm}-\rho_{00}\right) \sin ^{2} \gamma Q } \\
& \left.-\left[\rho_{ \pm \pm}\left(1+\cos ^{2} \gamma\right)+\rho_{00} \sin ^{2} \gamma\right] I_{0}\right\}
\end{aligned}
$$

$\frac{D Q}{D s}=-\frac{4 \pi^{3} d^{2} \omega_{r} \varphi_{\omega}}{h c}\left\{\left[\left(\rho_{b b}-\rho_{ \pm \pm}\right)\left(1+\cos ^{2} \gamma\right)+\left(\rho_{b b}-\rho_{00}\right) \sin ^{2} \gamma\right] Q-\left(\rho_{ \pm \pm}-\rho_{00}\right) \sin ^{2} \gamma I-\left(\rho_{ \pm \pm}-\rho_{00}\right) \sin ^{2} \gamma I_{0}\right\}$.

We define the two polarized intensities $I_{\perp}$ and $I_{\|}$by

$$
I_{\perp} \equiv \frac{1}{2}(I+Q), \quad I_{\|} \equiv \frac{1}{2}(I-Q),
$$

and rewrite equations (45) as

$$
\frac{D I_{q}}{D s}=-k_{q} \varphi_{\nu}\left[I_{q}-S_{q}\right], \quad q=\perp, \|
$$

where we have defined

$$
k_{\|}=\frac{4 \pi^{3} d^{2} \nu_{r}}{h c}\left\{\left(\rho_{b b}-\rho_{ \pm \pm}\right)\left[\left(1+\cos ^{2} \gamma\right) \pm \sin ^{2} \gamma\right]+\left(\rho_{b b}-\rho_{00}\right) \sin ^{2} \gamma(1 \mp 1)\right\}
$$

and

$$
S_{\|}=\frac{h \nu_{r}^{3}}{c^{2}}\left[\frac{8 \pi^{3} d^{2} \nu_{r}}{h c} \rho_{b b}-k \frac{\perp}{\|}\right] / k \frac{\perp}{\|}
$$

Thus we have recovered equations (1), (2), and (3) of Paper I.

\section{b) Case 2: Weak Field Limit}

In the limit of weak magnetic field, there is no preferred direction to take as the axis of quantization. We choose the principal axis $\hat{\boldsymbol{k}}$ to be the quantization axis. The polarization vectors $\hat{\boldsymbol{e}}_{\perp}$ and $\hat{\boldsymbol{e}}_{\|}=\hat{\boldsymbol{n}} \times \hat{\boldsymbol{e}}_{\perp}$ are chosen such that the Stokes parameter $U$ is zero. This requirement will be used to determine the angle $\eta$ between $\hat{\boldsymbol{e}}_{\perp}$ and $\hat{\boldsymbol{\varepsilon}}_{1}=(\hat{\boldsymbol{k}} \times \hat{\boldsymbol{n}}) /|\hat{\boldsymbol{k}} \times \hat{\boldsymbol{n}}|$. The circularly polarized vectors are $\hat{\boldsymbol{e}}^{ \pm}=\left(\hat{\boldsymbol{e}}_{\perp} \pm i \hat{\boldsymbol{e}}_{\|}\right) / \sqrt{2}$.

The matrix elements $V_{m b}$ are

$$
V_{m b}(s, t)=\frac{d}{\hbar}\left[\boldsymbol{E} \cdot \frac{(\hat{\boldsymbol{i}}-i \hat{\boldsymbol{j}})}{\sqrt{2}} \delta_{m,+}-\boldsymbol{E} \cdot \hat{\boldsymbol{k}} \delta_{m, 0}-\boldsymbol{E} \cdot \frac{(\hat{\boldsymbol{i}}+i \hat{\boldsymbol{j}})}{\sqrt{2}} \delta_{m,-}\right]
$$

The following relations hold:

$$
\begin{aligned}
\frac{\hat{\boldsymbol{i}} \pm i \hat{\boldsymbol{j}}}{\sqrt{2}} & =\exp ( \pm i \lambda)\left[\frac{(1 \pm \cos \vartheta)}{2} \exp (i \eta) \hat{\boldsymbol{e}}^{+}+\frac{(1 \mp \cos \vartheta)}{2} \exp (-i \eta) \hat{\boldsymbol{e}}^{-} \mp i \frac{\sin \vartheta}{\sqrt{2}} \hat{\boldsymbol{n}}\right] \\
\hat{\boldsymbol{k}} & =\cos \vartheta \hat{\boldsymbol{n}}-\frac{i \sin \vartheta}{\sqrt{2}}\left[\exp (i \eta) \hat{\boldsymbol{e}}^{+}-\exp (-i \eta) \hat{\boldsymbol{e}}^{-}\right]
\end{aligned}
$$


To derive expressions for the radiative rates $U_{ \pm \pm}, U_{00}, U_{ \pm \mp}$, and $U_{ \pm 0}$ we use definition (32) for $t=t^{\prime}$ and equations (10), (50), and (51). We obtain:

$$
\begin{gathered}
U_{ \pm \pm}(\omega)=\frac{\pi}{2 c}\left(\frac{d}{\hbar}\right)^{2}\left\{\left(1+\cos ^{2} \vartheta\right) I(\omega) \pm 2 \cos \vartheta V(\omega)+\sin ^{2} \vartheta[\cos 2 \eta Q(\omega)-\sin 2 \eta U(\omega)]\right\}, \\
U_{00}(\omega)=\frac{\pi}{c}\left(\frac{d}{\hbar}\right)^{2} \sin ^{2} \vartheta[I(\omega)-\cos 2 \eta Q(\omega)+\sin 2 \eta U(\omega)], \\
U_{ \pm \mp}(\omega)=-\frac{\pi}{2 c}\left(\frac{d}{\hbar}\right)^{2} \exp (\mp i 2 \lambda)\left\{\sin ^{2} \vartheta I(\omega)+\left(1+\cos ^{2} \vartheta\right)[\cos 2 \eta Q(\omega)-\sin 2 \eta U(\omega)]\right. \\
\mp i \cos \vartheta[\sin 2 \eta Q(\omega)+\cos 2 \eta U(\omega)]\}, \\
U_{ \pm 0}(\omega)= \pm i \frac{\pi}{c \sqrt{2}}\left(\frac{d}{\hbar}\right)^{2} \exp (\mp i \lambda) \sin \vartheta\{V(\omega) \pm \cos \vartheta I(\omega)+[i \sin 2 \eta \mp \cos \vartheta \cos 2 \eta] Q(\omega) \\
+[i \cos 2 \eta \pm \cos \vartheta \sin 2 \eta] U(\omega)\} .
\end{gathered}
$$

In what follows we set $V=0$ since circular polarization does not arise in our problem. The nonvanishing matrix elements of the dipole moment operator $\boldsymbol{d}=e r$ are given by

$$
d_{ \pm b}=\mp d \frac{(\hat{i} \mp i \hat{j})}{\sqrt{2}}, \quad d_{0 b}=d \hat{k} .
$$

Using equations (6), (40), (51), (53), and the rotating wave approximation for $\boldsymbol{P}$, we get

$$
\begin{aligned}
P^{ \pm}= & 2 d \int_{-\infty}^{\infty} \varphi_{v} d v\left\{-\frac{(1 \pm \cos \vartheta)}{2} \rho_{+b} \exp (i \lambda) \mp i \frac{\sin \vartheta}{\sqrt{2}} \rho_{0 b}+\frac{(1 \mp \cos \vartheta)}{2} \rho_{-b} \exp (-i \lambda)\right. \\
& \left.-\frac{(1 \mp \cos \vartheta)}{2} \rho_{+b}^{*} \exp (-i \lambda) \mp i \frac{\sin \vartheta}{\sqrt{2}} \rho_{0 b}^{*}+\frac{(1 \pm \cos \vartheta)}{2} \rho_{-b}^{*} \exp (i \lambda)\right\} \\
& \times \exp \left[i \omega_{r}\left(t-\frac{s}{c}\right)+i \varphi^{ \pm} \pm i \eta\right] .
\end{aligned}
$$

We express $E^{ \pm}$in terms of the $V$ 's using equations (3), (15), (50), and (51), and find

$$
E^{ \pm}=\left(\frac{\hbar}{d}\right)\left[V_{+b} \exp (i \lambda)-V_{-b} \exp (-i \lambda) \pm i \frac{\sqrt{2}}{\sin \vartheta} V_{0 b}\right] \exp \left[i \omega_{r}\left(t-\frac{s}{c}\right)+i \varphi^{ \pm} \mp i \eta\right] .
$$

Using equations (8), (9), (10), (21), (52), (54), and (55), we show that

$$
\begin{aligned}
\frac{D I}{D s}= & -\frac{4 \pi^{3} d^{2} \omega_{r} \varphi_{\omega}}{h c}\left\{\left(\rho_{b b}-\rho_{ \pm \pm}\right)\left[\left(1+\cos ^{2} \vartheta\right) I+\sin ^{2} \vartheta(\cos 2 \eta Q-\sin 2 \eta U)\right]\right. \\
& +\left(\rho_{b b}-\rho_{00}\right) \sin ^{2} \vartheta[I-(\cos 2 \eta Q-\sin 2 \eta U)] \\
& +\rho_{+-}\left[\cos 2 \lambda\left[\sin ^{2} \vartheta I+\left(1+\cos ^{2} \vartheta\right)(\cos 2 \eta Q-\sin 2 \eta U)\right]-2 \sin 2 \lambda \cos \vartheta(\sin 2 \eta Q+\cos 2 \eta U)\right] \\
& \left.-\left[\rho_{ \pm \pm}\left(1+\cos ^{2} \vartheta\right)+\rho_{00} \sin ^{2} \vartheta-\rho_{+-} \cos 2 \lambda \sin ^{2} \vartheta\right] I_{0}\right\}, \\
\frac{D Q}{D s}= & -\frac{4 \pi^{3} d^{2} \omega_{r} \varphi_{\omega}}{h c}\left\{\left(\rho_{b b}-\rho_{ \pm \pm}\right)\left[\left(1+\cos ^{2} \vartheta\right) Q+\cos 2 \eta \sin ^{2} \vartheta I\right]+\left(\rho_{b b}-\rho_{00}\right) \sin ^{2} \vartheta[Q-\cos 2 \eta I]\right. \\
& +\rho_{+-}\left[\cos 2 \lambda\left[\sin ^{2} \vartheta Q+\cos 2 \eta\left(1+\cos ^{2} \vartheta\right) I\right]-2 \sin 2 \lambda \sin 2 \eta \cos \vartheta I\right] \\
& \left.-\left[\left(\rho_{ \pm \pm}-\rho_{00}\right) \cos 2 \eta \sin ^{2} \vartheta-\rho_{+-} \cos 2 \lambda \cos 2 \eta\left(1+\cos ^{2} \vartheta\right)\right] I_{0}\right\},
\end{aligned}
$$




$$
\begin{aligned}
\frac{D U}{D s}=-\frac{4 \pi^{3} d^{2} \omega_{r} \varphi_{\omega}}{h c}\{ & \left(\rho_{b b}-\rho_{ \pm \pm}\right)\left[\left(1+\cos ^{2} \vartheta\right) U-\sin 2 \eta \sin ^{2} \vartheta I\right]+\left(\rho_{b b}-\rho_{00}\right) \sin ^{2} \vartheta[U+\sin 2 \eta I] \\
& +\rho_{+-}\left[\cos 2 \lambda \sin ^{2} \vartheta U-\cos 2 \lambda \sin 2 \eta\left(1+\cos ^{2} \vartheta\right) I-2 \sin 2 \lambda \cos 2 \eta \cos \vartheta I\right] \\
& \left.+\left[\left(\rho_{ \pm \pm}-\rho_{00}\right) \sin 2 \eta \sin ^{2} \vartheta-\rho_{+-} \cos 2 \lambda \sin 2 \eta\left(1+\cos ^{2} \vartheta\right)-2 \rho_{+-} \sin 2 \lambda \cos 2 \eta \cos \vartheta\right] I_{0}\right\}
\end{aligned}
$$

The angle $\eta$ is determined from equations (56). The terms in $D U / D s$ proportional to either $I$ or $I_{0}$ and the terms in $D I / D$ s proportional to $U$ sum to zero if

$$
\sin 2 \eta\left[\left(\rho_{ \pm \pm}-\rho_{00}\right) \sin ^{2} \vartheta-\rho_{+-} \cos 2 \lambda\left(1+\cos ^{2} \vartheta\right)\right]-\cos 2 \eta\left[2 \rho_{+-} \sin 2 \lambda \cos \vartheta\right]=0 .
$$

Furthermore, the terms in $D I / D s$ and $D Q / D s$ proportional to $Q$ and $I$, respectively, have as a factor

$$
\cos 2 \eta\left[\left(\rho_{ \pm \pm}-\rho_{00}\right) \sin ^{2} \vartheta-\rho_{+-} \cos 2 \lambda\left(1+\cos ^{2} \vartheta\right)\right]+\sin 2 \eta\left[2 \rho_{+-} \sin 2 \lambda \cos \vartheta\right]=K,
$$

which, after using equation (57) to eliminate $\eta$, becomes

$$
K=\left\{\left[\left(\rho_{ \pm \pm}-\rho_{00}\right) \sin ^{2} \vartheta-\rho_{+-} \cos 2 \lambda\left(1+\cos ^{2} \vartheta\right)\right]^{2}+\left[2 \rho_{+-} \sin 2 \lambda \cos \vartheta\right]^{2}\right\}^{1 / 2} .
$$

It will be convenient later on to use the following equivalent expressions for $K$ :

$$
K=-\frac{\left[\left(\rho_{00}-\rho_{ \pm \pm}\right) \sin ^{2} \vartheta+\rho_{+--} \cos 2 \lambda\left(1+\cos ^{2} \vartheta\right)\right]}{\cos 2 \eta}=\frac{2 \rho_{+-} \sin 2 \lambda \cos \vartheta}{\sin 2 \eta} .
$$

To avoid ambiguity we take $-\pi / 4 \leq \eta<\pi / 4$ so that $\cos 2 \eta \geq 0$. Then,

$$
\operatorname{sign}[K]=\operatorname{sign}\left[\left(\rho_{ \pm \pm}-\rho_{00}\right) \sin ^{2} \vartheta-\rho_{+-} \cos 2 \lambda\left(1+\cos ^{2} \vartheta\right)\right] .
$$

The radiative transfer equations now reduce to

$$
\begin{gathered}
\frac{D I}{D s}=-\frac{4 \pi^{3} d^{2} \omega_{r} \varphi_{\omega}}{h c}\left\{\left[\left(\rho_{b b}-\rho_{ \pm \pm}\right)\left(1+\cos ^{2} \vartheta\right)+\left(\rho_{b b}-\rho_{00}\right) \sin ^{2} \vartheta+\rho_{+-} \cos 2 \lambda \sin ^{2} \vartheta\right] I\right. \\
\left.-K Q-\left[\rho_{ \pm \pm}\left(1+\cos ^{2} \vartheta\right)+\rho_{00} \sin ^{2} \vartheta-\rho_{+-} \cos 2 \lambda \sin ^{2} \vartheta\right] I_{0}\right\} \\
\frac{D Q}{D s}=-\frac{4 \pi^{3} d^{2} \omega_{r} \varphi_{\omega}}{h c}\left\{\left[\left(\rho_{b b}-\rho_{ \pm \pm}\right)\left(1+\cos ^{2} \vartheta\right)+\left(\rho_{b b}-\rho_{00}\right) \sin ^{2} \vartheta+\rho_{+-} \cos 2 \lambda \sin ^{2} \vartheta\right] Q-K I-K I_{0}\right\}
\end{gathered}
$$

We define the two polarized intensities $I_{\perp}$ and $I_{\|}$by

$$
I_{\perp} \equiv \frac{1}{2}(I+Q), \quad I_{\|} \equiv \frac{1}{2}(I-Q),
$$

and rewrite equations (52) and (53) as follows:

$$
\frac{D I_{q}}{D s}=-k_{q} \varphi_{\nu}\left[I_{q}-S_{q}\right], \quad q=\perp, \|,
$$

where

$$
\begin{aligned}
k_{\frac{\perp}{\|}}= & \frac{4 \pi^{3} d^{2} \nu_{r}}{h c}\left\{\left(\rho_{b b}-\rho_{ \pm \pm}\right)\left[\left(1+\cos ^{2} \vartheta\right) \pm \cos 2 \eta \sin ^{2} \vartheta\right]+\left(\rho_{b b}-\rho_{00}\right) \sin ^{2} \vartheta(1 \mp \cos 2 \eta)\right. \\
& \left.+\rho_{+-}\left[\cos 2 \lambda\left[\sin ^{2} \vartheta \pm \cos 2 \eta\left(1+\cos ^{2} \vartheta\right)\right] \mp 2 \sin 2 \lambda \sin 2 \eta \cos \vartheta\right]\right\},
\end{aligned}
$$


and

$$
S_{\frac{1}{\|}}=\frac{h \nu_{r}^{3}}{c^{2}}\left[\frac{8 \pi^{3} d^{2} \nu_{r}}{h c}-k_{\Perp}\right] / k_{\|}
$$

The expressions (66) and (67) for the absorption coefficients and source functions reduce to the corresponding strong field expressions (48) and (49) if we set $\rho_{+-}=0$ and take the magnetic field direction along $\hat{k}$.

\section{SOBOLEV APPROXIMATION}

In the Sobolev approximation the profile averaged specific intensity inside the source is given by (Castor 1970)

$$
\int_{0}^{\infty} d \nu \varphi_{\nu} I_{q}(\nu, \hat{\boldsymbol{n}}, \boldsymbol{r})=S_{q}(\boldsymbol{r}, \hat{\boldsymbol{n}})-\left[S_{q}(\boldsymbol{r}, \hat{\boldsymbol{n}})-\frac{B}{2}\right] \beta_{q}(\boldsymbol{r}, \hat{\boldsymbol{n}})
$$

Here the external radiation field is assumed to be due entirely to the cosmic blackbody radiation $B$ and $\beta_{q}(\boldsymbol{r}, \hat{\boldsymbol{n}})$, the escape probability in direction $\hat{\boldsymbol{n}}$ at position $\boldsymbol{r}$, is given by

$$
\beta_{q}(\boldsymbol{r}, \hat{\boldsymbol{n}})=\frac{\left[1-\exp \left(-\tau_{q}\right)\right]}{\tau_{q}}
$$

with $\tau_{q}(\nu, \boldsymbol{r}, \hat{\boldsymbol{n}})$ the total optical depth at frequency $\nu$ along the ray $\hat{\boldsymbol{n}}$ which passes through $\boldsymbol{r}$. The frequency $\nu$ is related to the velocity $v(r)$ by $\nu=\nu_{0}(1-\hat{n} \cdot v / c)$. The optical depth varies inversely with $|\hat{n} \cdot \nabla v(r)|$ and is

$$
\tau_{q}(\nu, \boldsymbol{r}, \hat{\boldsymbol{n}})=\frac{c k_{q}(\boldsymbol{r}, \hat{\boldsymbol{n}})}{\nu_{0}\left|\sum_{i} \Lambda_{i}(\boldsymbol{r}) n_{i}^{2}\right|},
$$

where $n_{i}$ are the components of $\hat{\boldsymbol{n}}$ along the principal axes of the symmetric part of the rate-of-strain tensor.

Radio astronomers measure the difference between the true specific intensity and the cosmic blackbody radiation. Thus the appropriate definition of polarization $P$ is

$$
P=\frac{I_{\perp}^{\text {excess }}-I_{\|}^{\text {excess }}}{I_{\perp}^{\text {excess }}+I_{\|}^{\text {excess }}}
$$

with

$$
I_{q}^{\text {excess }}=I_{q}-\frac{1}{2} B=\left(S_{q}-\frac{1}{2} B\right)\left[1-\exp \left(-\tau_{q}\right)\right]
$$

\section{ASYMPTOTIC EXPRESSIONS FOR THE POLARIZATION}

The equations of motion of the density matrix and the equations of radiative transfer may be solved analytically in the limits of low and high optical depth under conditions in which all of the $\Lambda_{i}$ have the same sign. These conditions correspond to pure expansion $\left(\Lambda_{i}>0\right)$ or pure contraction $\left(\Lambda_{i}<0\right)$ together with an arbitrary rotation. Our ultimate goal is to write down analytic expressions for the polarization. In what follows, we only state our procedure and quote our results.

\section{a) Case 1: Strong Field Limit}

The strong field limit was presented in Paper I. For completeness we simply quote the final results.

The asymptotic expressions for the polarization are the following: For TAU $\ll 1$

$$
P=\frac{3}{40} \sin ^{2} \gamma \operatorname{TAU}[1-f(\alpha, \beta)]\left\{1+\frac{10}{3} \frac{\left(C+3 C^{\prime}\right)}{A}\left[1-\exp \left(-\frac{h \nu_{r}}{k T_{\mathrm{BB}}}\right)\right]\right\}^{-1}
$$


and for $\mathrm{TAU} \gg 1$,

$$
P=\frac{\sin ^{2} \gamma}{2 \mathrm{TAU}}[1-f(\alpha, \beta)]\left[1+\frac{10}{3} \frac{\left(C+3 C^{\prime}\right)}{A} \frac{\left(\rho_{b b}-\rho_{a a}\right)}{\rho_{b b}}\right]^{-1},
$$

where TAU is the "mean" optical depth defined by

$$
\mathrm{TAU}=\frac{24 \pi^{3} d^{2}\left(\rho_{b b}-\rho_{a a}\right)}{h\left|\Lambda_{1}+\Lambda_{2}+\Lambda_{3}\right|},
$$

$\rho_{a a}$ is the average population of the upper sublevels, i.e., $\rho_{a a}=\left(2 \rho_{ \pm \pm}+\rho_{00}\right) / 3, T_{\mathrm{BB}}$ is the cosmic blackbody temperature,

$$
f(\alpha, \beta)=\frac{3\left[\sin ^{2} \alpha\left(\Lambda_{1} \sin ^{2} \beta+\Lambda_{2} \cos ^{2} \beta\right)+\Lambda_{3} \cos ^{2} \alpha\right]}{\Lambda_{1}+\Lambda_{2}+\Lambda_{3}}
$$

and for TAU $\gg 1$,

$$
\frac{\left(\rho_{b b}-\rho_{a a}\right)}{\rho_{b b}}=1-\left\{\frac{C}{A} \exp \left(-\frac{h \nu_{r}}{k T}\right)+\frac{1}{\mathrm{TAU}} \frac{\exp \left(-h \nu_{r} / k T_{\mathrm{BB}}\right)}{\left[1-\exp \left(-h \nu_{r} / k T_{\mathrm{BB}}\right)\right]}\right\}\left\{\frac{C}{A}+\frac{1}{\mathrm{TAU}} \frac{1}{\left[1-\exp \left(-h \nu_{r} / k T_{\mathrm{BB}}\right)\right]}\right\}^{-1} .
$$

The angles $\alpha$ and $\beta$ determine the direction of the magnetic field, which is given by

$$
\hat{b}=(\sin \alpha \sin \beta, \sin \alpha \cos \beta, \cos \alpha) \text {. }
$$

There is an extra requirement for the validity of equation (73), namely

$$
\frac{3\left|\Lambda_{i}-\Lambda_{j}\right|}{\left|\Lambda_{1}+\Lambda_{2}+\Lambda_{3}\right|} \ll 1
$$

\section{b) Case 2: Weak Field Limit}

The procedure we follow is essentially similar to that outlined in Paper I for the strong field limit. First, we evaluate analytically the rates $R_{ \pm \pm}, R_{00}, R_{+-}, A_{ \pm \pm}$, and $A_{00}$. We do this by using definitions (33) and expressions (52). In expressions (52) we set $V=U=0, I=I_{\perp}+I_{\|}, Q=I_{\perp}-I_{\|}, I_{q}=\int_{-\infty}^{\infty} I_{q}(\nu) \varphi_{\nu} d \nu$, and use equations (68), (69), and (67). We set $\rho_{00}=\rho_{ \pm \pm}+\Delta \rho$ and keep terms up to first order in $\Delta \rho_{,} \rho_{+-}$, and TAU or $1 /$ TAU depending on whether we are in the low or high optical depth limit, respectively. Second, we substitute the radiative rates into equations (35) and solve for $\Delta \rho$ and $\rho_{+-}$under steady state conditions. Finally, we evaluate the polarization $P$ after expanding equation (71) to first order and using the expressions for $\Delta \rho$ and $\rho_{+-}$derived above. In what follows we state the results of the above three steps in the limits of low and high optical depth TAU.

i) Case 2a: High Optical Depth

In this limit the radiative rates are given by

$$
\begin{aligned}
& R_{ \pm \pm}=g\left\{1+\frac{1}{10} \frac{\rho_{b b}}{\rho_{ \pm \pm}} \frac{\Delta \rho}{\left(\rho_{b b}-\rho_{ \pm \pm}\right)}-\frac{3}{10 \mathrm{TAU}}\left[\frac{3 \Lambda_{1}+3 \Lambda_{2}+4 \Lambda_{3}}{\Lambda_{1}+\Lambda_{2}+\Lambda_{3}}\right]\left[1-\frac{\left(\rho_{b b}-\rho_{ \pm \pm}\right)}{\rho_{ \pm \pm}\left[\exp \left(h \nu_{r} / k T_{\mathrm{BB}}\right)-1\right]}\right]\right\} \\
& R_{00}=g\left\{1+\frac{4}{5} \frac{\rho_{b b}}{\rho_{ \pm \pm}} \frac{\Delta \rho}{\left(\rho_{b b}-\rho_{ \pm \pm}\right)}-\frac{3}{10 \mathrm{TAU}}\left[\frac{4 \Lambda_{1}+4 \Lambda_{2}+2 \Lambda_{3}}{\Lambda_{1}+\Lambda_{2}+\Lambda_{3}}\right]\left[1-\frac{\left(\rho_{b b}-\rho_{ \pm \pm}\right)}{\rho_{ \pm \pm}\left[\exp \left(h \nu_{r} / k T_{\mathrm{BB}}\right)-1\right]}\right]\right\} \\
& R_{+-}=g\left\{\frac{7}{10} \frac{\rho_{b b}}{\rho_{ \pm \pm}} \frac{\rho_{+-}}{\left(\rho_{b b}-\rho_{ \pm \pm}\right)}+\frac{3}{10 \mathrm{TAU}} \frac{\left(\Lambda_{2}-\Lambda_{1}\right)}{\left(\Lambda_{1}+\Lambda_{2}+\Lambda_{3}\right)}\left[1-\frac{\left(\rho_{b b}-\rho_{ \pm \pm}\right)}{\rho_{ \pm \pm}\left[\exp \left(h \nu_{r} / k T_{\mathrm{BB}}\right)-1\right]}\right]\right\} \\
& R_{ \pm 0}=0
\end{aligned}
$$


where

$$
g=A \frac{\rho_{ \pm \pm}}{\left(\rho_{b b}-\rho_{ \pm \pm}\right)} .
$$

Note that our assumption that $R_{ \pm 0}=0$ in $\S$ III is now shown to be self-consistent.

The expressions for $\Delta \rho=\rho_{00}-\rho_{ \pm \pm}$and $\rho_{+-}$are given by

$$
\begin{gathered}
{\left[1+\frac{10}{3} \frac{\left(\rho_{b b}-\rho_{a a}\right)}{\rho_{b b}} \frac{\left(C+3 C^{\prime}\right)}{A}\right]\left(\rho_{ \pm \pm}-\rho_{00}\right)} \\
=\frac{1}{\operatorname{TAU}} \frac{\left(\rho_{b b}-\rho_{a a}\right)}{\rho_{b b}} \frac{\left[\rho_{a a}-\rho_{b b} \exp \left(-h \nu_{r} / k T_{\mathrm{BB}}\right)\right]}{\left[1-\exp \left(-h \nu_{r} / k T_{\mathrm{BB}}\right)\right]} \frac{\left(\Lambda_{1}+\Lambda_{2}-2 \Lambda_{3}\right)}{\left(\Lambda_{1}+\Lambda_{2}+\Lambda_{3}\right)} \\
\rho_{+-}=\left(\rho_{ \pm \pm}-\rho_{00}\right) \frac{\left(\Lambda_{2}-\Lambda_{1}\right)}{\left(\Lambda_{1}+\Lambda_{2}-2 \Lambda_{3}\right)}
\end{gathered}
$$

We see that $\Delta \rho, \rho_{+-}$, and $1 /$ TAU are all of the same order, as assumed earlier in this section.

The polarization $P$ is given by

$$
\begin{aligned}
P= & \frac{1}{2 \operatorname{TAU}} \frac{\operatorname{sign}[K]}{\left(\Lambda_{1}+\Lambda_{2}+\Lambda_{3}\right)} \\
& \times \frac{\left\{4\left(\Lambda_{2}-\Lambda_{1}\right)^{2} \cos ^{2} \vartheta \sin ^{2} 2 \lambda+\left[\left(2 \Lambda_{3}-\Lambda_{1}-\Lambda_{2}\right) \sin ^{2} \vartheta+\left(\Lambda_{2}-\Lambda_{1}\right)\left(1+\cos ^{2} \vartheta\right) \cos 2 \lambda\right]^{2}\right\}^{1 / 2}}{\left\{1+(10 / 3)\left[\left(\rho_{b b}-\rho_{a a}\right) / \rho_{b b}\right]\left(C+3 C^{\prime}\right) / A\right\}},
\end{aligned}
$$

where $\left(\rho_{b b}-\rho_{a a}\right) / \rho_{b b}$ is given by equation (76b). If $\Lambda_{1}=\Lambda_{2}$, expression (81) reduces to expression (74) with the magnetic field taken along $\hat{\boldsymbol{k}}$. This is expected because for $\Lambda_{1}=\Lambda_{2}, \rho_{+-}=0$.

ii) Case 2b: Low Optical Depth

Here the radiative rates are given by

$$
\begin{aligned}
R_{ \pm \pm} & =A\left\{\frac{1}{\left[\exp \left(h \nu_{r} / k T_{\mathrm{BB}}\right)-1\right]}+\frac{\mathrm{TAU}}{20}\left(11-f^{\prime}\right)\left[\frac{\rho_{a a}}{\left(\rho_{b b}-\rho_{a a}\right)}-\frac{1}{\left[\exp \left(h \nu_{r} / k T_{\mathrm{BB}}\right)-1\right]}\right]\right\} \\
R_{00} & =A\left\{\frac{1}{\left[\exp \left(h \nu_{r} / k T_{\mathrm{BB}}\right)-1\right]}+\frac{\mathrm{TAU}}{10}\left(4+f^{\prime}\right)\left[\frac{\rho_{a a}}{\left(\rho_{b b}-\rho_{a a}\right)}-\frac{1}{\left[\exp \left(h \nu_{r} / k T_{\mathrm{BB}}\right)-1\right]}\right]\right\} \\
R_{+-} & =A \frac{3}{20} \mathrm{TAU} \frac{\left(\Lambda_{2}-\Lambda_{1}\right)}{\left(\Lambda_{1}+\Lambda_{2}+\Lambda_{3}\right)}\left[\frac{\rho_{a a}}{\left(\rho_{b b}-\rho_{a a}\right)}-\frac{1}{\left[\exp \left(h \nu_{r} / k T_{\mathrm{BB}}-1\right)\right]}\right] \\
R_{ \pm 0} & =0
\end{aligned}
$$

where

$$
f^{\prime} \equiv \frac{3 \Lambda_{3}}{\Lambda_{1}+\Lambda_{2}+\Lambda_{3}}
$$

The expressions for $\Delta \rho=\rho_{00}-\rho_{ \pm \pm}$and $\rho_{+-}$are

$$
\begin{gathered}
\left(\rho_{ \pm \pm}-\rho_{00}\right)=\frac{3 \mathrm{TAU}}{20}\left(1-f^{\prime}\right) \frac{\left[\rho_{a a}-\rho_{b b} \exp \left(-h \nu_{r} / k T_{\mathrm{BB}}\right)\right]}{\left\{1+\left[\left(C+3 C^{\prime}\right) / A\right]\left[1-\exp \left(-h \nu_{r} / k T_{\mathrm{BB}}\right)\right]\right\}} \\
\rho_{+-}=\frac{\left(\Lambda_{2}-\Lambda_{1}\right)}{\left(\Lambda_{1}+\Lambda_{2}-2 \Lambda_{3}\right)}\left(\rho_{ \pm \pm}-\rho_{00}\right)
\end{gathered}
$$




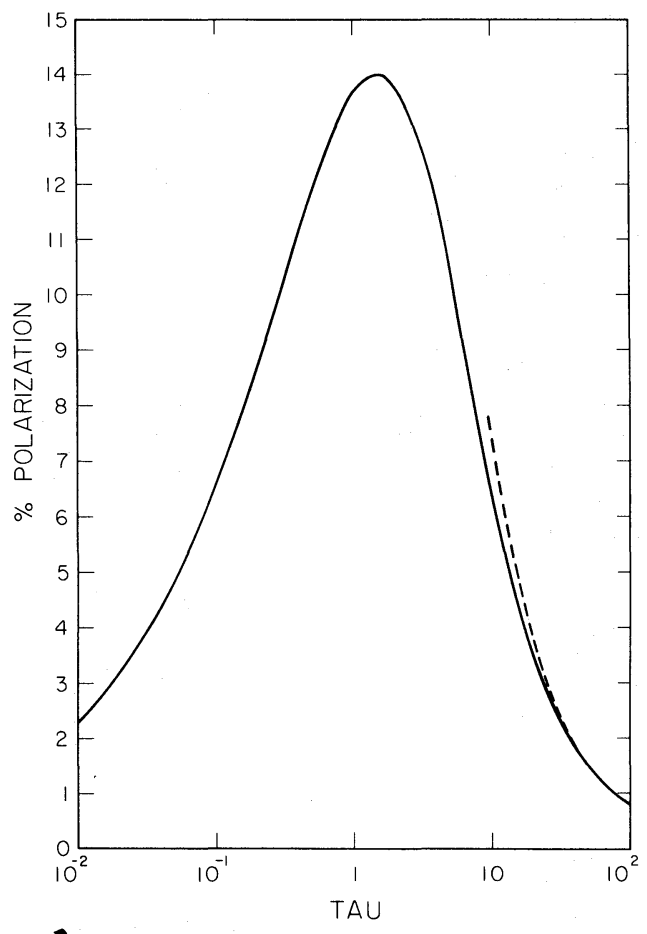

FIG. 1.-Polarization as a function of optical depth TAU. The solid line gives our numerical results, while the dashed one corresponds to the asymptotic expressions (76) and (83). The values of the parameters used are: $\Lambda_{1}=\Lambda_{2}=0, \Lambda_{3}=1 \times 10^{-11} \mathrm{~s}^{-1}, \lambda_{r}=c / \nu_{r}=0.3 \mathrm{~cm}$, $\left(C+3 C^{\prime}\right) / A=0.212, T=10 \mathrm{~K}, T_{\mathrm{BB}}=2.7 \mathrm{~K}, \vartheta=\pi / 2$, and $\lambda=0$.

Finally, the asymptotic expression for the polarization $P$ reads

$$
\begin{aligned}
P= & \frac{\text { TAU }}{40} \frac{\operatorname{sign}[K]}{\left(\Lambda_{1}+\Lambda_{2}+\Lambda_{3}\right)} \\
& \times \frac{\left\{4\left(\Lambda_{2}-\Lambda_{1}\right)^{2} \cos ^{2} \vartheta \sin ^{2} 2 \lambda+\left[\left(2 \Lambda_{3}-\Lambda_{1}-\Lambda_{2}\right) \sin ^{2} \vartheta+\left(\Lambda_{2}-\Lambda_{1}\right)\left(1+\cos ^{2} \vartheta\right) \cos 2 \lambda\right]^{2}\right\}^{1 / 2}}{\left\{1+\left[\left(C+3 C^{\prime}\right) / A\right]\left[1-\exp \left(-h \nu_{r} / k T_{\mathrm{BB}}\right)\right]\right\}} .
\end{aligned}
$$

As in the strong field case, equation (85) holds under the condition

$$
\frac{3\left|\Lambda_{i}-\Lambda_{j}\right|}{\left|\Lambda_{1}+\Lambda_{2}+\Lambda_{3}\right|} \ll 1
$$

To determine the polarization $P$ for TAU near unity, one must numerically integrate the rate equations (34) in the case of a strong magnetic field, or equations (35) in the case of a weak magnetic field. The radiative rates are calculated from definitions (33) using the Sobolev approximation.

We analyze a specific example of a molecular cloud undergoing one-dimensional collapse, where the directions of collapse and magnetic field lie along $\hat{k}$ and the observation direction in the $(\hat{\boldsymbol{k}}, \hat{\boldsymbol{i}})$-plane. Thus $\Lambda_{1}=\Lambda_{2}=0$, and $\Lambda_{3} \neq 0$. The results are shown in Figure 1. Since $\Lambda_{1}=\Lambda_{2}, \rho_{+-}=0$ and there is no difference between the weak and strong field limits. The polarization is proportional to $\sin ^{2} \vartheta$, and its maximum value is 0.14 . If we note that $(1-f)=-2$ in this example, we can easily compute $P$ for several other configurations. For example, if we keep everything else the same, but take the magnetic field along $\hat{\boldsymbol{j}},(1-f)=1$ and the polarization $P$ is positive and its magnitude is equal to $\frac{1}{2}$ of that shown in Figure 1. 
VII. DISCUSSION

Since the observed line width is several times the thermal width, different portions of the line come from different regions in the source. Thus, it is to be expected that the magnitude of the polarization, and perhaps its direction as well, may vary across the line.

Our assumption that the systematic velocity differences in the source are much greater than the thermal velocities of the molecules is not a necessary condition for the existence of polarization. Polarization arises in a static medium if the optical depth is anisotropic. In fact, inhomogeneous (Doppler) broadening dominates for interstellar radio and far infrared lines. Thus, even in a static medium the escape of photons from a region of high optical depth is predominantly by frequency diffusion rather than spatial diffusion. In such a case, the Sobolev approximation is not valid. However, the radiative transfer may, to a good approximation, be described by a version of the escape probability formalism (Athay and Skumanich 1971).

We are indebted to Charles Alcock, Fred Lo, and Tom Phillips for helpful advice. This research was supported by NSF grants AST80-20005 and AST79-22012. This is contribution number 3613 from the Division of Geological and Planetary Sciences, California Institute of Technology, Pasadena, California 91125.

Athay, R. G., and Skumanich, A. 1971, Ap. J., 170, 605.

Castor, J. I. 1970, M.N.R.A.S., 149, 111.

Chandrasekhar, S. 1950, Radiative Transfer (Oxford: Clarendon Press).

Goldreich, P., Keeley, D. A., and Kwan, J. Y. 1973, Ap. J., 179, 111

Goldreich, P., and Kylafis, N. D. 1981, Ap. J. (Letters), 243, L75 (Paper I).

Peter Goldreich: Division of Geological and Planetary Sciences 170-25, California Institute of Technology, Pasadena, CA 91125

Nikolaos D. Kylafis: Theoretical Astrophysics 130-33, California Institute of Technology, Pasadena, CA 91125 